\title{
Prenatal Diagnosis and Clinical Course of a Patent Urachus Associated with an Allantoic Cord Cyst and a Giant Umbilical Cord
}

\section{Introduction}

Umbilical cord cysts are rare and the various differential diagnoses influence both management of delivery and postnatal care. Differential diagnoses of umbilical cord cysts include pseudo-cysts, omphalocele, bladder exstrophy, omphalomesenteric duct cysts and other urachal anomalies. Communication between the cyst and the fetal bladder is a hallmark of patent ductus urachus, which has an estimated incidence of 1-2:100,000 and may be detected prenatally by high-resolution ultrasound (Persutte WH et al. J Ultrasound Med 1988; 7: 399-403; Wildhaber BE et al. Arch Dis Child Fetal Neonatal Ed 2005; 90: F535536). We present a case of a patent ductus urachus initially presenting as an allantoic cord cyst followed by edematous swelling of the umbilical cord.

\section{Case Description}

A 33-year-old woman, gravida 1 para 0 , was referred to our medical practice for detailed ultrasound (US) examination of an umbilical cord cyst detected during a routine antenatal scan. Her previous medical and ob- stetric history was unremarkable. At 22 weeks of gestation (WG), ultrasound examination showed a live singleton fetus with biometrical data consistent with gestational age and an amniotic fluid volume in the upper normal range. The presence of an isolated, fluid-filled umbilical cyst $(26 \times 28 \times 28 \mathrm{~mm})$ close to the fetal insertion site was confirmed ( $\triangleright$ Fig. 1a). Communication between the cyst and the fetal bladder was not evident, the urogenital tract, intestinal tract and the abdominal wall appeared normal. At 26 WG, an umbilical-urachal sinus was visible ( $>$ Fig. 1 $\mathbf{b}$ ) and at 29 WG the umbilical cord was clearly edematous (max. diameter $43 \mathrm{~mm}$, not shown). However, umbilical cord perfusion was not impaired as was shown by Doppler sonography. At 33 WG, a connection between the urachus and the fetal bladder could be demonstrated ( $\triangleright$ Fig. 1c), supporting the suspicion of a patent urachus. Whereas the umbilical cord remained edematous ( $>$ Fig. 2 ), the size of the cyst decreased during the last weeks of gestation and had nearly resolved at 36+4 WG ( $\triangleright$ Fig. 1c, d). Fetal development progressed normally and at 40 WG, a male in- fant (4,390 g) was spontaneously delivered. The placenta appeared normally developed with a size of $22 \times 19.3 \times 3 \mathrm{~cm}$ and a weight of $650 \mathrm{~g}$. Fetal membranes were unremarkable. The umbilical cord was $60 \mathrm{~cm}$ in length and showed pronounced and widespread edema with a max. diameter of $6 \mathrm{~cm}$. Histopathological examination revealed intense edema of the Wharton's jelly ( $>$ Fig. 3).

During the first $24 \mathrm{~h}$ of life repeated leakage of urine from the umbilical cord stump was noted, confirming the diagnosis of a patent urachus. During the following two days, no dribbling of urine was observed and the stump seemed to become sclerotic. At 3 days of life a sonographic examination showed a partially filled bladder with a funnel-shaped structure emerging at its dome and leading $2-3 \mathrm{~cm}$ towards the navel. However, it could not be followed to the abdominal wall. The patient was returned home and a further examination was scheduled on day 7 . Since repeated leakage of urine from the navel was observed during the following days, the patent urachus was surgically excised at day 9 and the dome of the bladder closed. Histological examination of the surgical speci-
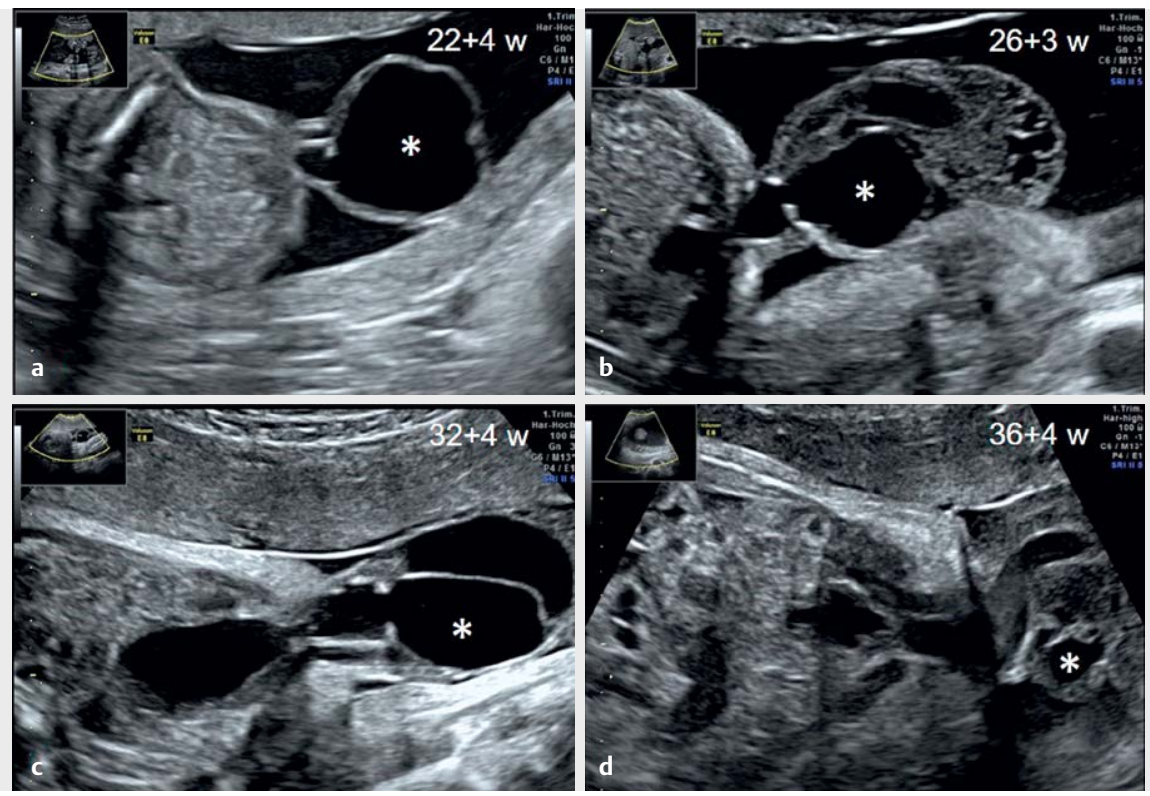

- Fig. 1 Development of the allantoic cyst during pregnancy: as gestation progressed, the umbilical cord cyst decreased in size and resolved. ( ${ }^{*}$ allantoic cyst). 


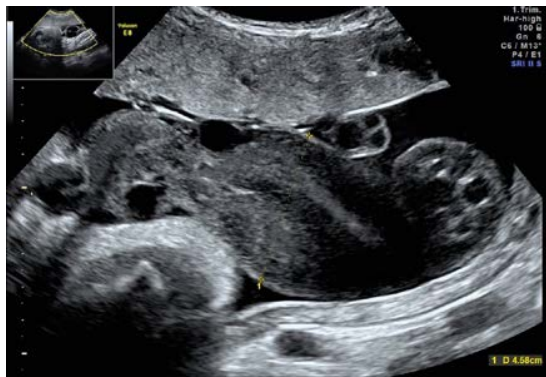

-Fig. 2 2D ultrasound image of edematous Wharton's jelly with cystic appearance, probably resulting from urine absorption into the umbilical cord (longitudinal view, 33 weeks of gestation).

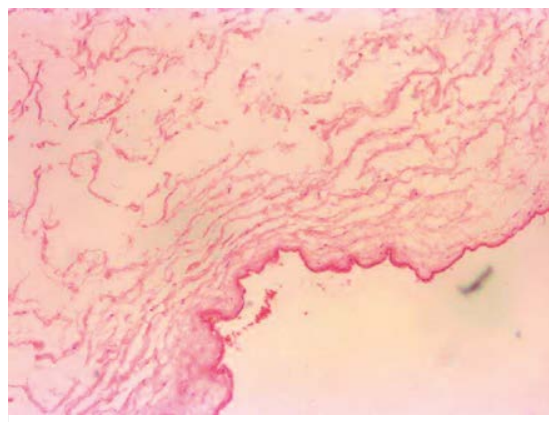

- Fig. 3 Histopathologic section of the Wharton's jelly with edematous degeneration.

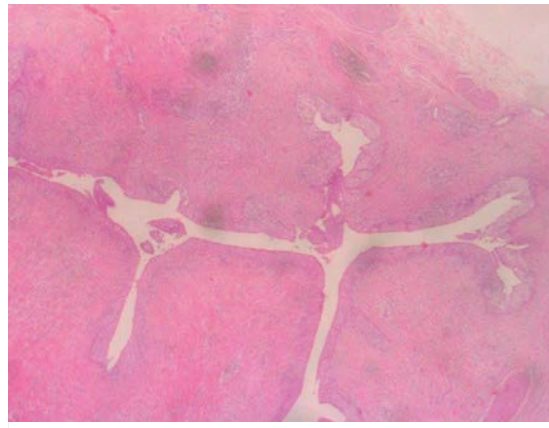

-Fig. 4 Histological examination of the surgical specimen depicts the patent urachus lined with transitional epithelium.

men confirmed the diagnosis ( $\triangleright$ Fig. 4). Postoperative progress was uneventful.

\section{Discussion}

In the presented case, a prominent isolated anechoic umbilical cyst close to the fetal insertion site was detected at week 22 of ges- tation. Close monitoring was initiated and at week 33 direct communication of the allantoic cyst with the fetal bladder could be established, supporting the diagnosis of a patent urachus. The size of the allantoic cyst was markedly decreased at week 36. Previous cases describing allantoic cysts associated with patent urachus similarly describe shrinkage of umbilical cysts during the last weeks of gestation, probably due to the draining of fluid through its connection to the bladder (Persutte WH et al. JUItrasound Med 1988; 7: 399-403; Rasteiro C et al. Ultrasound Obstet Gynecol 2013; 42: 239-240;). However, the fate of the cyst appears to vary since reports describe either sudden disappearance due to putative rupture (Umeda S et al. Eur J Pediatr Surg 2016; 26: 200-202) or increase of the cyst size (Wildhaber BE et al. Arch Dis Child Fetal Neonatal Ed 2005; 90: F535-536).

As gestation progressed, we observed edematous thickening of the umbilical cord and the infant was delivered with a prominent giant umbilical cord. Only a few cases of such edematous umbilical cords with an extraordinarily large diameter have been described in the literature in association with a patent urachus and it is thought that its development is due to the absorption of urine into the Wharton's jelly (Wildhaber BE et al. Arch Dis Child Fetal Neonatal Ed 2005; 90: F535-536; Haac B et al. Pediatr Surg Int 2017; 33: 393-395; Schiesser M et al. Ultrasound Obstet Gynecol 2003; 22: 646-647).

In our case, due to the confirmation of communication between the bladder and the umbilicus, the diagnosis of a patent urachus was already most likely prenatally. No other abdominal wall defects or urinary tract anomalies were noted. An isolated patent urachus is usually not associated with aneuploidies (Haac B et al. Pediatr Surg Int 2017; 33: 393-395). Nevertheless, on prenatal detection of a patent urachus, close monitoring of the mother and fetus should be initiated in order to rule out fetal compromise and in order to evaluate the appropriate birth mode. Wildhaber et al., described a case where repeated fetal bradycardia after onset of labor, possibly induced by compression of the umbilical vessels by the cystic mass, necessitated an emergency Caesarean section (Wildhaber
BE et al. Arch Dis Child Fetal Neonatal Ed 2005; 90: F535-536). In our case, the cysts had almost resolved at term and spontaneous birth progressed without complications. Postnatally, the diagnosis of the patent urachus was obvious because leakage of urine from the umbilicus was noted. Spontaneous closure did not occur, and surgical intervention was necessary. Among the few cases of patent urachus reported in the literature, the majority needed surgical repair which usually has an excellent prognosis.

\section{Conflict of Interest}

The authors declare that they have no conflict of interest.

\section{Authors}

Ismail Tekesin ${ }^{1}$, Regina Küper-Steffen ${ }^{2}$

\section{Affiliations}

1 Prenatal Unit Stuttgart, Prenatal Unit Stuttgart, Stuttgart, Germany

2 Klinikum Stuttgart, Department of Pathology, Stuttgart, Germany

\section{Correspondence}

\author{
PD Dr. Ismail Tekesin \\ Prenatal Unit Stuttgart \\ Alte Poststrasse 3 \\ 71073 Stuttgart \\ Germany \\ Tel.: + 49-711-4006010 \\ Fax: +49-711-4006010 \\ tekesin@praenatalstuttgart.de
}

\section{Bibliography}

DOI https://doi.org/10.1055/a-0633-3879 Ultrasound Int Open 2018; 4: E104-E105 (c) Georg Thieme Verlag KG Stuttgart . New York ISSN 2199-7152

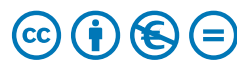

